\title{
Effect of "Modular" Nursing Intervention on the Therapeutic Effect of Ranoxifene on Postmenopausal Osteoporosis
}

\author{
GUOYang ${ }^{1}$, SunXiaoJie ${ }^{2}$, TaiMiao $^{2}$, LiuFang $^{1}$, LiuXiaoWei $^{1}$, YaoJie $^{1 *}$ \\ ${ }^{1}$ College of Nursing, Shaanxi University of traditional Chinese Medicine, xianyang 712000, China \\ ${ }^{2}$ Affiliated hospital of shaanxi university of Chinese medicine, xianyang 712000 , China
}

\begin{abstract}
: the purpose of this study was to explore the effect of "modular" nursing intervention on Ranoxifene in the treatment of patients with postmenopausal osteoporosis. A total of 108 patients with postmenopausal osteoporosis who were accepted by the Department of Orthopaedics in our hospital were selected as the research objects. According to the random number table method and they were divided into two groups (the intervention group and the control group), 54 cases in each group. The control group was given routine care, while the other was given "modular" interventional care. The patient satisfaction, adverse mood changes and quality of life changes in the two groups was observed in the treatment. The results of this study showed that the nursing satisfaction of the patients in the intervention group was significantly higher than that in the control group; however, the negative emotion of the two groups of patients after care were lower than that before the care, and the degree of reduction in the intervention group was significantly higher than that of the control group; the life quality of patient in the two groups was improved than before nursing, and the degree of improvement in the intervention group was significantly higher than that in the control group. Therefore, the clinical application of Raloxifene in the treatment of postmenopausal osteoporosis patients with "modular" nursing intervention can improve patient satisfaction, relieve negative emotions, and improve quality of life.
\end{abstract}

\section{1 introduction}

Osteoporosis (OP) is a systemic bone disease characterized by low bone mass and destruction of bone microstructure, which leads to increased bone fragility and prone to fracture. The attack of osteoporosis is closely related to age, and estrogen deficiency is the main cause of postmenopausal osteoporosis [1] Menopause is an important transition period in the physiological process of women. Most women have ovarian failure and atrophy after menopause, with great reduction of estrogen. A large amount of bone loss often manifests as bone pain, spinal deformity, multiple organ dysfunction and fractures, which seriously affects the life quality of patients [2]. In the nursing process of postmenopausal osteoporosis patients, clinical nurses tend to easily ignore the assessment of patients' negative mood and poor quality of life. Therefore, this study introduced the "modular" education model [3] into clinical nursing to provide nursing care. In terms of content, it is the nursing content led by the nursing goals that are of the same nature or internally connected to form a relatively independent nursing unit. All nursing units should proceed from the overall nursing goal and design each nursing unit based on the nursing goal content. It is a relatively "complete nursing" process in which the nursing measures of each unit play roles in a comprehensive way. The implementation of "modular" nursing intervention in the research achieved good results, and the reports are as follows.

\section{Data and Method}

\subsection{General data}

From July to December 2019, 108 patients with postmenopausal osteoporosis treated with Ranoxifene were selected and divided into the intervention group and the control group according to random number table method, 54 cases in each group. The patients of the intervention group aged between 50 and 70 , with an average age of $60.80 \pm 5.67$ years old; the bone mineral density in this group was $-0.4--3.4\left(\mathrm{~g} / \mathrm{cm}^{3}\right)$, with an average of $-1.87 \pm 0.91\left(\mathrm{~g} / \mathrm{cm}^{3}\right) ; 8$ of these patients were with an educational background of senior high school and 46 with junior high school or below. The patients of the control group aged between 51 and 72, with an average age of $62.06 \pm 5.20$ years old; the bone mineral density in this group was $-0.4--3.6(\mathrm{~g} / \mathrm{cm} 3)$, with an average of $-1.96 \pm 0.90(\mathrm{~g} / \mathrm{cm} 3) ; 10$ of these patients were with an educational background of senior high school, and 44 with junior high school or below. There was no statistically significant difference between the two groups

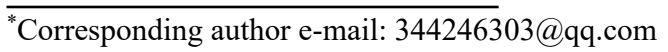


in terms of age, bone density, education level and other general data $(\mathrm{P}>0.05)$, and thus they were comparable.

Inlusion criteria: (1) Those who clinically met the diagnostic criteria for postmenopausal osteoporosis; (2) Those who were $\geq 50$ years old and had been menopausal for $\geq 1$ year; (3) Those who suffered obvious spontaneous bone pain; (4) Those without thromboembolism before admission; (5) Those who gave informed consent and agreed to accept the nursing model for nursing.

Exclusion criteria:(1) Those who did not meet the clinical diagnostic criteria for postmenopausal osteoporosis; (2) Those with secondary osteoporosis, severe liver and kidney dysfunction, severe cardiovascular and cerebrovascular diseases, tumors, endocrine, and rheumatism affecting bone metabolism diseases; (3) Those who had other drugs for the treatment of osteoporosis within a month; (4) Those who had thromboembolism before admission.

\subsection{Method}

Patients in the control group took routine care measures after admission to the hospital, including introduction of disease-related knowledge, medication guidance, dietary guidance, support and encouragement for patients and their families, and so on.

Patients in the intervention group were given "modular" nursing intervention based on routine nursing:

(1) Cognitive intervention. Nursing staff should introduce the causes and common clinical manifestations of postmenopausal osteoporosis to patients and their family. The estrogen level of middle-aged women decreases with age. On the one hand, estrogen can directly affect bone tissue, inhibit bone turnover and promote bone densification [4]; on the other hand, when estrogen is deficient, osteoclast function is active, bone resorption increases and bone formation reduces. Therefore, estrogen deficiency may be the main cause of osteoporosis in postmenopausal women. Raloxifene is a selective estrogen receptor modulator (SERMs), which acts as an estrogen agonist in the skeletal system. After binding to the estrogen receptor, the spatial conformation of the receptor is changed in different target tissues, thereby exerting similar or antagonistic different biological effects of estrogen in different tissues [5], which can not only inhibit the bone resorption of osteoclasts, but also promote the osteogenic effect of osteoblasts. Related systematic review has proved that the drug Raloxifene present good safety. Compared with Alendronate, the two have no significant difference in reducing fracture risk, incidence of adverse events and upper gastrointestinal adverse events [6]; however, the incidence of venous thrombosis is high, and thus this drug is prohibited for those with a history of venous thrombosis and a tendency to thrombosis.

(2) Pharmacological care. In addition to the protective effect of estrogen on bone and cardiovascular,
Raloxifene can fight estrogen in the endometrium and breast, so it does not increase the risk of cancer. The drug is taken orally at an early stage of menopause, $60 \mathrm{mg} / \mathrm{d}$. It can be taken at any time of the day and is not restricted by meals. Nursing staff should urge patients to regularly test urine calcium and bone density content, and patients without adverse reactions can take it for a long time. The fracture risk rate can be reduced by $30 \%-50 \%$ after $4-31$ weeks of maintenance. Raloxifene is mainly metabolized in the liver, and thus this drug is not recommended for patients with hepatic insufficiency. If the patient suffers from symptoms of liver dysfunction after taking it, the doctor in charge should be notified to closely detect whether serum total bilirubin, g-glutamyl transaminase, alkaline phosphatase, ALT and AST are elevated. It has been found in clinical application that if patients are accompanied by hypertriglyceridemia $(5.6 \mathrm{mmol} / \mathrm{L})$, the use of Raloxifene may cause a further increase in their serum triglyceride levels, so serum triglyceride levels should be monitored when patients with such a history take Raloxifene.

(3) Behavioral intervention. Nursing staff should help patients establish a healthy lifestyle and intervene in their unhealthy behavior, such as persuading them to avoid drinking, excessive coffee, excessive smoking and overeating. It is also necessary to inform them to increase the amount of outdoor exercise and vitamin D supplementation in a proper amount, besides a balanced and reasonable diet. In addition to arranging the patients' life in a reasonable manner, the nursing staff should also tell them to insist on taking the medicine and ask them to report back in time for adverse reactions and seek medical treatment.

(4) Psychological nursing. Nursing staff should strengthen the psychological nursing of patients and explain in detail the relationship between their emotions and diseases, so that they can realize the importance of maintaining good emotions. It is important to communicate with patients and their family to create a warm atmosphere for medical treatment and maintain a relaxed attitude. In the course of daily care, nursing staff can strengthen communication with patients and guide them to vent their bad emotions. Family members can also be involved in daily nursing work, so that patients can fully feel the warmth. When patients suffer from bad emotions, nursing staff should listen and guide patiently to reduce anxiety and keep them calm.

\subsection{Observation indexes}

(1) The nursing satisfaction scale adopted in this study was based on the nursing satisfaction questionnaire designed by Han Qi et al [7], and it had been modified according to the specific conditions of the department. The overall coefficient of Cronbach's $\alpha$ is 0.812 , and the difference is statistically significant. The satisfaction is divided into three levels: unsatisfied, neutral and satisfied. (Unsatisfied $\leq 5$ points; 6 points $\leq$ neutral $\leq 18$ points; satisfied $\geq 19$ points)

(2) The evaluation standard of bad emotions includes the Anxiety Self-Assessment Scale (SAS) and Depression 
Self-Assessment Scale (SDS). Both scales include 20 items, and each item has a score ranges between 1 and 4 . The critical score of SDS scale is 53 points, and that of SAS scale is 50 points. The higher the score is, the more severe the anxiety and depression [8].

(3) The quality of life score is evaluated according to the World Health Organization Quality of Life Scale (WHOQOL-100), including physical, psychological, social relations, environmental and spiritual pillars and independence. The higher the score is, the higher the quality of life [9].

\subsection{Statistical treatment}

SPSS19.0 software was used for data entry and statistical processing. The measurement data was expressed as $(\mathrm{x} \pm \mathrm{s})$ and the deduction employed $\mathrm{X} 2$ test, $\mathrm{t}$ test and $\mathrm{z}$ test. $\mathrm{P}<0.05$ indicated that the difference was statistically significant.

\section{Results}

\subsection{Comparison of nursing satisfaction between two groups}

The nursing satisfaction of the patients in the intervention group is $89 \%$, which is significantly higher than that of the control group $(78 \%) . P<0.05$, the difference is statistically significant, as shown in Table 1.

Table 1 Comparison of patient nursing satisfaction ( $\mathrm{x} \pm \mathrm{s}$, unit: case)

\begin{tabular}{lccccc}
\hline Group & $\mathrm{N}$ & Dissatisfied & More satisfied & satisfaction & Always satisfied \\
\hline Control group & 54 & 12 & 31 & 11 & 42 \\
Intervention group & 54 & 6 & 26 & 22 & 48 \\
$\mathrm{Z}$ & & & & & -2.44 \\
$\mathrm{P}$ & & & & 0.02 \\
\hline
\end{tabular}

\subsection{Comparison of adverse emotions between the two groups}

There is no significant difference in the SAS and SDS scores between the two groups of patients before nursing
$(\mathrm{P}>0.05)$. The SAS and SDS scores of the two groups after nursing are significantly lower than before nursing, and the intervention group is significantly lower than the control group. The difference is statistically significant $(\mathrm{P}$ $<0.05$ ), as shown in Table 2

Table 2 Comparison of patients' bad ē̄otions ( $\mathrm{x} \pm \mathrm{s}$, unit: $\min )$

\begin{tabular}{|c|c|c|c|c|c|}
\hline \multirow[t]{2}{*}{ Group } & \multirow[t]{2}{*}{$\mathrm{N}$} & \multicolumn{2}{|c|}{ Before nursing } & \multicolumn{2}{|c|}{ After nursing } \\
\hline & & SAS score & SDS score & SAS score & SDS score \\
\hline Control group & 54 & $56.04 \pm 2.63$ & $57.06 \pm 2.61$ & $42.07 \pm 3.03$ & $44.94 \pm 4.11$ \\
\hline Intervention group & 54 & $55.00 \pm 2.87$ & $57.04 \pm 2.68$ & $36.50 \pm 4.03$ & $43.39 \pm 4.06$ \\
\hline $\mathrm{T}$ & & 1.95 & 0.04 & -6.29 & -6.37 \\
\hline $\mathrm{P}$ & & 0.06 & 0.98 & 0.00 & 0.00 \\
\hline
\end{tabular}

\subsection{Comparison of quality of life scores between the two groups}

There is no significant difference in the total quality of life scores of the two groups of patients before nursing $(\mathrm{P}>0.05)$. The quality of life scores of the two groups of patients after nursing are significantly improved compared with that before nursing and the degree of improvement in the intervention group is significantly higher than that of the control group. The difference is statistically significant $(\mathrm{P}<0.05)$.

Table 3 Comparison of patients' quality of life scores ( $\mathrm{x} \pm \mathrm{s}$, unit: points)

\begin{tabular}{lccc}
\hline Group & $\mathrm{N}$ & Before nursing & After nursing \\
\hline Control group & 54 & $26.56 \pm 2.31$ & $36.74 \pm 2.70$ \\
Intervention group & 54 & $26.41 \pm 2.51$ & $45.11 \pm 4.05$ \\
$\mathrm{Z}$ & & -0.22 & -7.81 \\
$\mathrm{P}$ & & 0.83 & 0.00 \\
\hline
\end{tabular}

\section{Discussion}

As a selective estrogen receptor agonist, Raloxifene is the most commonly used drug in the treatment of osteoporosis in clinic. Related animal experiments have shown that the drug has a similar effect to estrogen. It only slightly inhibits bone transformation and has little effects on the activity of osteoblasts [10], and thus it is 
more suitable for the treatment of postmenopausal osteoporosis. It is clearly stated in 2018 Chinese Guideline for the Diagnosis and Treatment of Senile Osteoporosis that Raloxifene can significantly increase the bone density of the lumbar spine and hip and reduce the risk of new vertebral fractures, the overall incidence of adverse events and the incidence of new nonvertebral fracture. According to Camacho PM et al (2016), Raloxifene can be used as the best grade of initial medication for patients who need to improve vertebral body disease [11]. In 2017, it was pointed out in Brazilian Rheumatism Association: Guidelines for the Diagnosis and Treatment of Postmenopausal Osteoporosis: Raloxifene $(60 \mathrm{mg} / \mathrm{d})$ can be used to prevent and treat women patients who suffer postmenopausal osteoporosis without menopausal symptoms, and it can significantly reduce the risk of vertebral fractures [12]. In 2017, the British National Osteoporosis Guidelines Group (NOGG): British Osteoporosis Clinical Prevention and Treatment Guidelines stated that: Raloxifene replacement therapy can be used for those who are intolerant or contraindications to estrogen drugs [13].

It is can be found from the analysis of this study that it is feasible to implement "modular" nursing intervention for postmenopausal osteoporosis patients treated with Ranoxifene in clinical practice, which can improve clinical nursing satisfaction. The data show that that the nursing satisfaction of the intervention group was $89 \%$, significantly higher than the control group (78\%), and the difference is statistically significant (P $<0.05)$. Therefore, it proved the superiority of the four "modular" nursing interventions, namely, cognitive intervention, pharmacological care, behavioral intervention and psychological nursing. The implementation of cognitive interventions and behavioral interventions can improve patients' awareness of the disease, so that they can better cooperate with the nursing staff in the treatment. Based on the clear understanding of the drug mechanism and contraindications in detail, nursing staff can formulate drug care plans according to the individual differences of patients, which greatly improves the safety of medication. The data also showed that the SAS and SDS score of patients in the intervention group was significantly lower than those in the control group. Nursing staff encourage patients to vent their anxiety, which can better improve their psychological conditions and reduce their stress. At the same time, nursing staff can provide psychological care according to the education level of patients, which can better improve their bad mood [14-15]. The results of this study showed that the quality of life scores of the two groups of patients after care were significantly improved compared with those before the treatment, and the improvement of the intervention group was higher than that of the control group; and the difference was statistically significant $(\mathrm{P}<0.05)$. Thus, if the nursing process is specifically divided into 4 independent modules and a nursing plan is developed according to the nursing focus of each module to carry out targeted care, the treatment will have better clinical effects. This treatment method can actively mobilize patients' life initiative and make them receive treatment better, which can greatly improve their quality of life.

To sum up, the implementation of Ranoxifene with "modular" nursing intervention for patients with postmenopausal osteoporosis can improve their clinical nursing satisfaction, alleviate their negative emotions and improve treatment compliance. At the same time, it can also improve the quality of their life by helping them develop a healthy and optimistic attitude.

\section{References}

1. Ma Yuanzheng, Wang Yipeng, Liu Qiang, Chinese Journal of Gerontology.J, 39 (2019).

2. Wu Sanmei, Jiang Quanhong, Yang Liuqing, China Medical Herald .J, 12 (2015).

3. Li Xiangrui, Hu Asia, Chinese Journal of Medical Education.J, 39 (2019).

4. Wang Jianhua, Chinese Journal of Geriatric Orthopedics and Rehabilitation.J, 5 (2019).

5. Um MJ, Cho EA, Jung H, J Menopausal Med.J, 23 (2017).

6. Lin T, Yan SG, Cai XZ, Int J Endocrinol.J,78(2014).

7. Han Qi, Xu Chen, Wang Ronghua, Nursing Research.J, 14 (2019).

8. Okumus M, Ceceli E, Tasbas O, J Back Musculoskelet Rehabil.J, 26 (2013).

9. Wang Lihua, Liu Hao, Inner Mongolian Traditional Chinese Medicine.J, 33 (2014).

10. Taranta A, Brama M, Teti A, Bone.J, 30 (2002).

11. Camacho PM, Petak SM, Binkley N, Endocr Pract.J, 22 (2016).

12. Radominski SC, Bernardo W, Paula AP, Rev Bras Re-umatol (Engl Ed).J, 57 (2017).

13. Nayak S, Roberts MS, Greenspan SL. Ann Intern Med.J, 155 (2011).

14. Zhi Wenyan, Zhang Huijuan, Ji Fengmin, Nursing Research.J, 23 (2009).

15. Zeng Guohua, International Journal of Nursing.J, 31 (2012). 